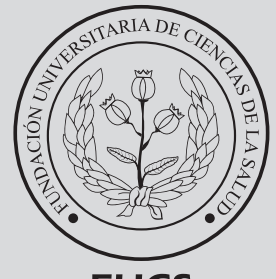

FUCS
Repro pertan

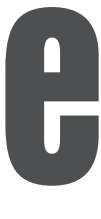

\title{
Lesiones de la raíz posterior de los meniscos
}

Gustavo A. Rincón MD Eiber Sanabria MD $^{\mathbf{b}}$

\section{Posterior meniscal root tears}

${ }^{a}$ Servicio de Ortopedia Hospital de San José, Fundación Universitaria de Ciencias de la Salud. Bogotá DC, Colombia.

${ }^{b}$ Ortopedia y Traumatología, Fundación Universitaria de Ciencias de la Salud. Bogotá DC, Colombia.

\section{R E S U M E N}

Las raíces son fundamentales para la función meniscal debido a que son el punto de anclaje en el platillo tibial y permiten la adecuada función de los mismos. Sus lesiones deben ser motivo de alta sospecha diagnóstica para evitar daños condrales precoces. El tratamiento debe ser la reinserción de la lesión ya que el seguimiento a corto plazo ha demostrado buenos resultados. El trasplante meniscal está reservado como cirugía de salvamento cuando no es posible realizar el reparo.

Palabras clave: menisco, raíz, reinserción, lesión.

(C) 2020 Fundación Universitaria de Ciencias de la Salud - FUCS Este es un artículo Open Access bajo la licencia CC BY-NC-ND (http://creativecommons.org/licenses/by-nc-nd/4.0/).

\section{ABSTRACT}

The meniscal roots are essential for their proper functioning. They are the meniscal anchorage point into the tibial plateau maintaining their normal function. Diagnosis of these lesions requires a high degree of suspicion to prevent early development of chondral injuries. Surgical root repair should be attempted, for short-term follow-up has evidenced good outcomes. Meniscal transplantation is reserved for salvage situations when a meniscus cannot be repaired.

Key words: meniscus, root, repair, tear.

C 2020 Fundación Universitaria de Ciencias de la Salud - FUCS This is an open access article under the CC BY-NC-ND license (http://creativecommons.org/licenses/by-nc-nd/4.0/).

Historia del artículo:

Fecha recibido: mayo 2 de 2019 Fecha aceptado: septiembre 18 de 2019

\section{INFORMACIÓN DEL ARTÍCULO}

\footnotetext{
Autor para correspondencia.

Dr. Gustavo A. Rincón

garincon@fucsalud.edu.co
}

DOI

10.31260/RepertMedCir.01217273.971 


\section{IN T RODUCCIÓN}

Las lesiones de la raíz posterior de los meniscos no fueron entidades reconocidas durante mucho tiempo, a pesar de que existen algunos reportes de avulsiones meniscales con fragmento óseo que datan de 1935. Fue el grupo de Pagnani y col. ${ }^{1}$ quienes las describieron en 1991 en un caso de menisco interno en un joven atleta. A pesar de tener diagnóstico, la mayoría de casos fueron tratados mediante meniscectomía parcial debido a que no se sabían reparar. Así mismo, no se entendía la gravedad de la misma y la utilidad de fijar el menisco lesionado. La aparición de la resonancia magnética fue de gran utilidad para reconocer esta entidad y fue sin duda una excelente herramienta para diagnosticarla y crear estrategias de tratamiento. La mayoría de la literatura disponible hace énfasis en el menisco medial, sin embargo todos esos conceptos también son aplicables en las lesiones del menisco lateral. ${ }^{2-4}$

\section{EPIDEMIOLOGÍA}

Existe una distribución bimodal en las lesiones de la raíz de los meniscos, ${ }^{5,6}$ un primer pico aparece en pacientes que han presentado un trauma en la tercera década de vida alrededor de los 22 años. Se asocian con rupturas del ligamento cruzado anterior y por lo regular ocurren en el cuerno posterior del menisco lateral. También se han asociado con lesiones multiligamentarias. El segundo pico ocurre en la sexta década de la vida alrededor de los 55 años y casi siempre obedecen a causas degenerativas aisladas, en pacientes con sobrepeso. Las lesiones de la raíz posterior del menisco medial tienen como factores de riesgo el sexo femenino, un alto índice de masa corporal y bajo nivel de actividad física. ${ }^{7}$ Otras lesiones de la raíz se presentan con mayor frecuencia en hombres que practican deportes de impacto, que ante el compromiso de la raíz del menisco lateral existe un alto riesgo de ruptura asociada del ligamento cruzado anterior (OR 10,3 IC95\% 2,62, 42,5). ${ }^{8}$ En los hombres hay mayor riesgo de severidad (OR 7.38 IC $95 \%$ $1,92,27,61){ }^{9}$

\section{PRESENTACIÓN CLÍNICA Y DIAGNÓSTICO}

La forma más común de presentación clínica de una lesión de la raíz meniscal es muy parecida a la del cuerno posterior de los meniscos, sin embargo la aparición es de inicio súbito con dolor más severo y típicamente se encuentra un dolor en la interlínea posteromedial o posterolateral, según sea el caso. ${ }^{10,11} \mathrm{Al}$ examen físico se halla efusión articular, limitación para la flexión completa y signo de McMurray positivo. Las radiografías suelen ser normales excepto cuando ocurre una avulsión con fragmento óseo, lo que simularía un "osículo meniscal". ${ }^{10,12,13}$ La resonancia magnética es fundamental en el diagnóstico, ofrece una sensibilidad entre 68 y 100\% y es bien conocido el signo del "menisco fantasma" por ausencia de la inserción del menisco en los cortes sagitales. ${ }^{13,14}$ También se puede apreciar la amputación abrupta de la raíz posterior en los cortes coronales así como una lesión radial en la misma vista. Pero sin duda, la más importante es la extrusión meniscal de más de $3 \mathrm{~mm}$ en los cortes coronales, al nivel del tercio medio del ligamento colateral medial. Si se presenta una extrusión meniscal de 3 o más milímetros, puede existir una lesión de la raíz o adyacente a la raíz hasta en $88 \%$ de los casos; sin embargo, si es menor de $3 \mathrm{~mm}$ solo se verá en $3 \%$ de los mismos y seguramente serán lesiones radiales mayores de $50 \%$ o degenerativas con varios planos de clivaje $^{13-16}$ (figuras 1 a 3).

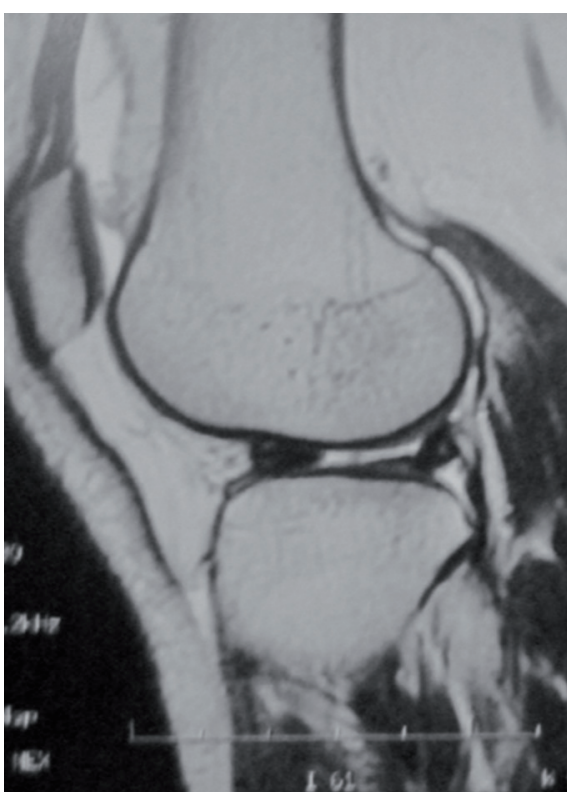

Figura 1. Signo de menisco fantasma.

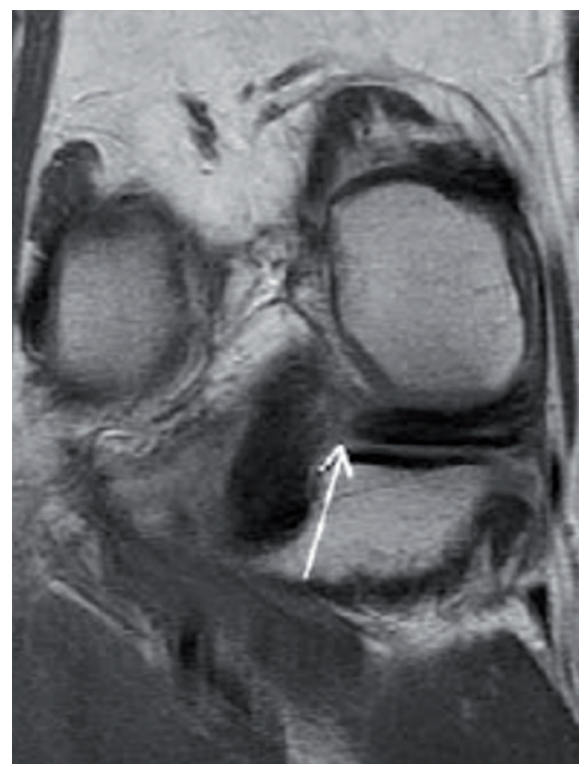

Figura 2. Amputación de la raíz. 


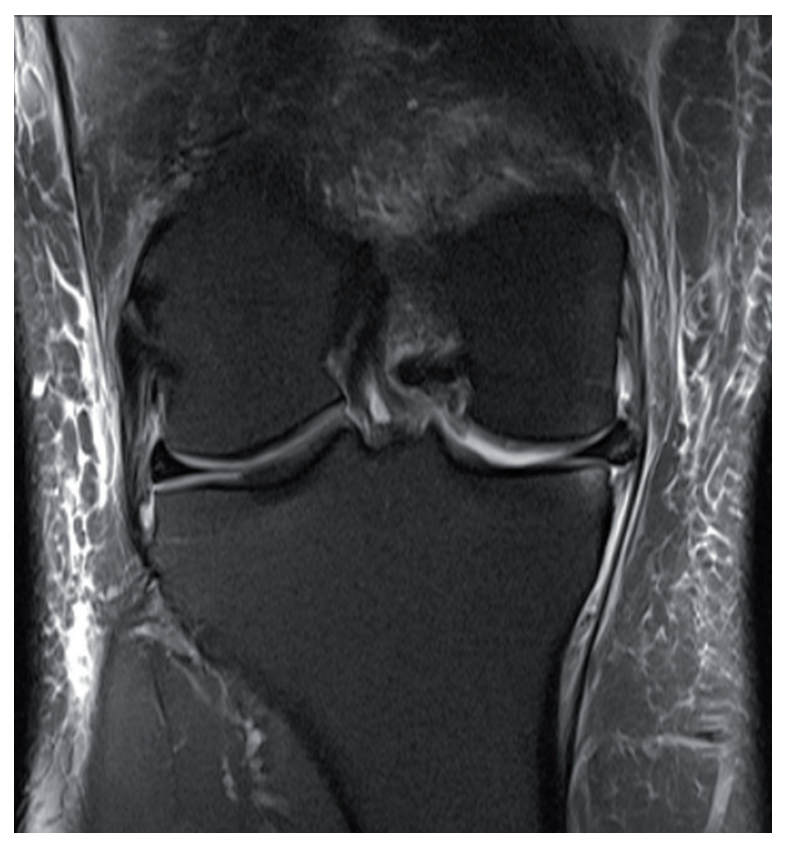

Figura 3. Extrusión meniscal.

A pesar de ser una excelente herramienta diagnóstica, la resonancia magnética presenta limitaciones; como no se realiza con apoyo de la extremidad, la extrusión se puede subestimar; es posible que se altere en los casos con efusión articular, en las lesiones en asa de balde concomitante y en los pacientes artrósicos con osteofitos tibiales. Por esta razón en el servicio de ortopedia del Hospital de San José de Bogotá consideramos que el diagnóstico debe basarse en la clínica, la resonancia magnética y los hallazgos de la artroscopia. $^{15,16}$

\section{CLASIFICACIÓN}

No hay consenso en la literatura con respecto a una clasificación que nos describa los tipos de lesión de la raíz posterior de los meniscos. El grupo de la Universidad de Pittsburgh (West - Kim - Harner), ${ }^{6,11}$ la definió como la lesión que ocurre a $1 \mathrm{~cm}$ de la inserción ósea del mismo y lo clasificó de la siguiente manera: (figuras 4 a 6): tipo I: avulsiones de la raíz; tipo II: lesiones radiales próximas a la raíz y tipo III: lesiones complejas.

LaPrade $^{17}$ propuso también un sistema de clasificación en 5 tipos diferentes (figura 7): tipo 1: lesión parcial meniscal dentro de 0 a $9 \mathrm{~mm}$ de la raíz meniscal; tipo 2: lesión radial completa; se describen tres subtipos: a. lesión radial completa de 0 a $3 \mathrm{~mm}$ de la inserción, b. lesión radial completa de 3 a $6 \mathrm{~mm}$ de la inserción y c. lesión radial completa de 6 a $9 \mathrm{~mm}$ de la lesión; tipo 3: lesión en asa de balde con desprendimiento completo de la raíz; tipo 4: lesión oblicua compleja que se extiende a la inserción de la raíz y tipo 5: fractura por avulsión de la inserción de la raíz meniscal.

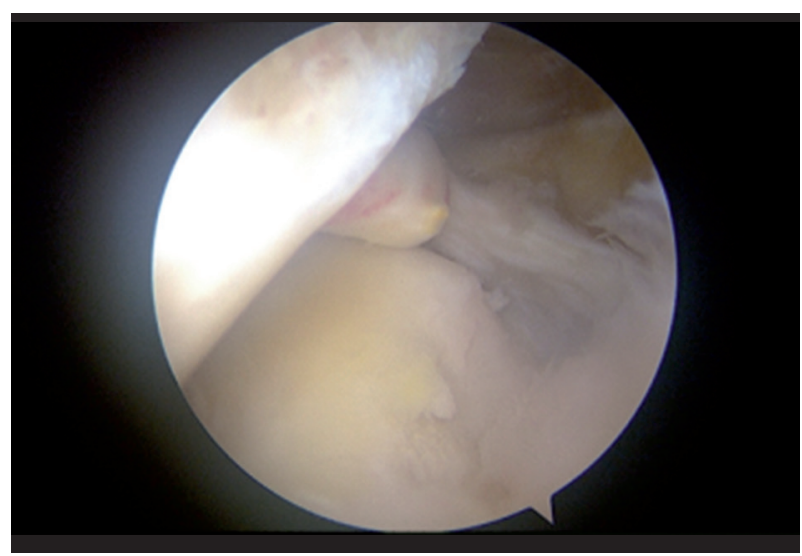

Figura 4. Tipo I: avulsiones de la raíz

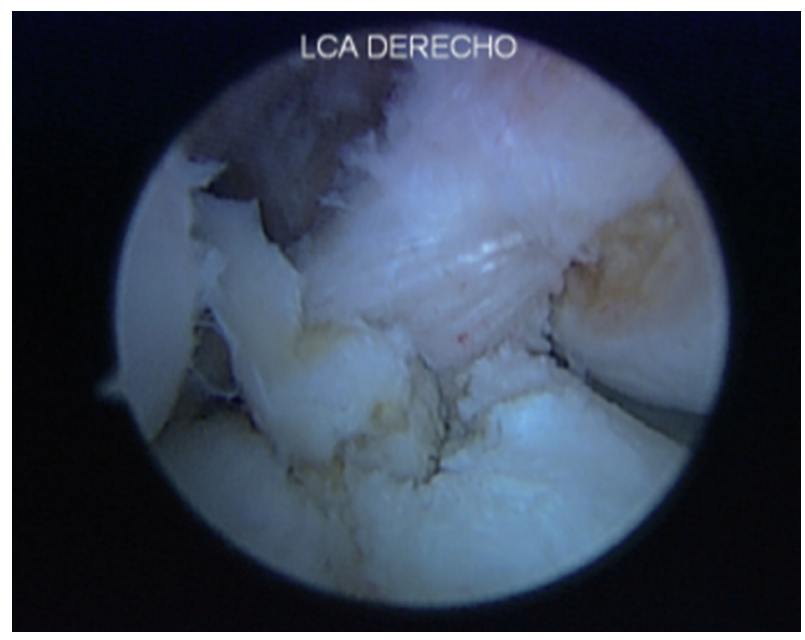

Figura 5. Tipo II. lesiones radiales próximas a la raíz.

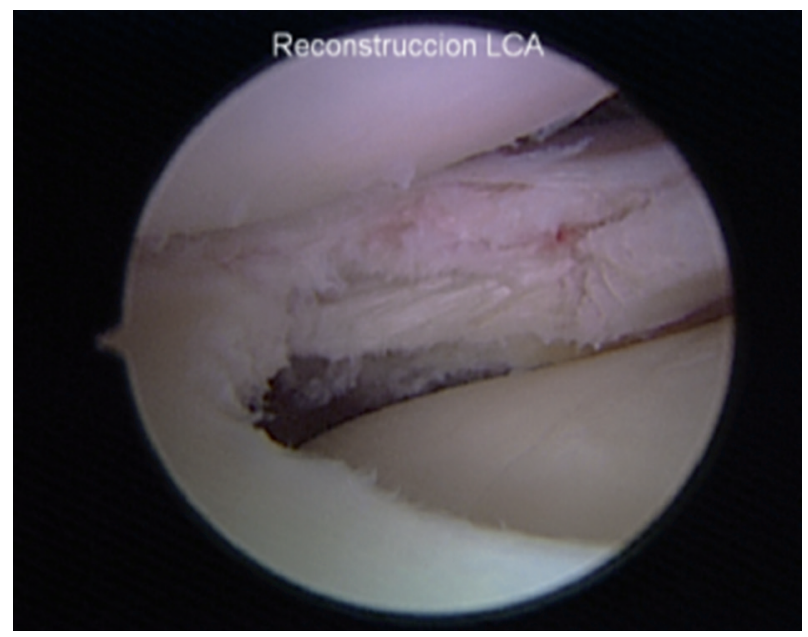

Figura 6. Tipo III lesiones complejas. 


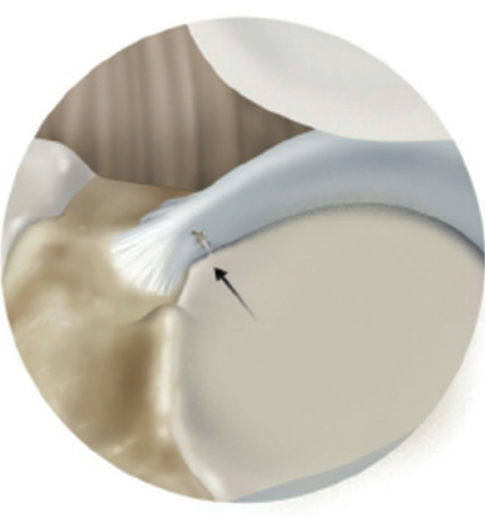

Tipo 1

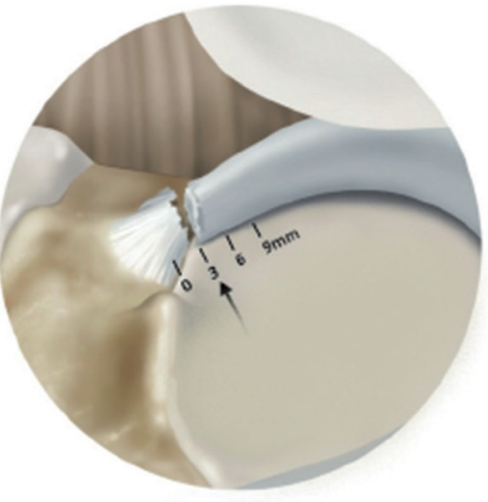

Tipo 2

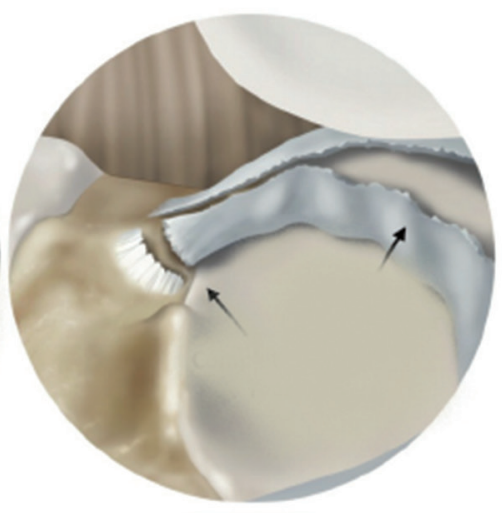

Tipo 3

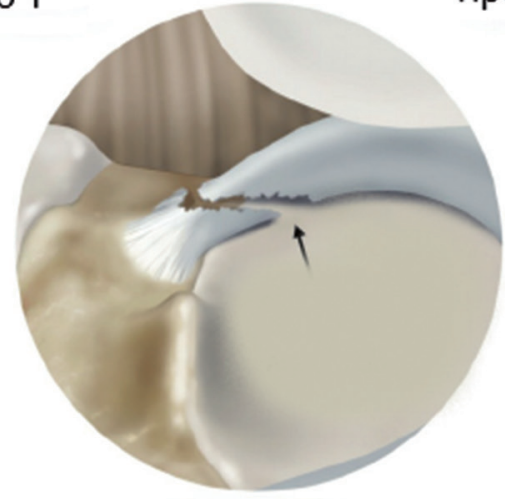

Tipo 4

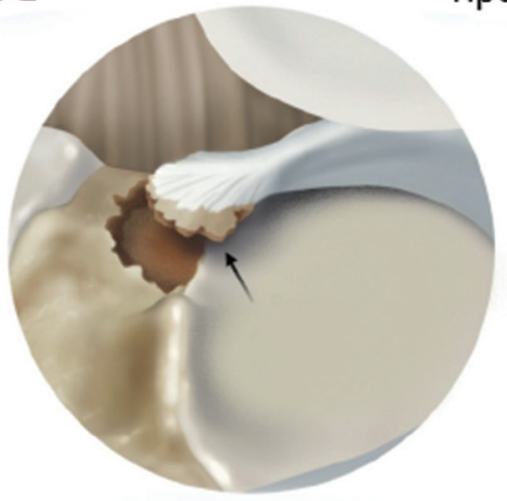

Tipo 5

Figura 7. Clasificación de LaPrade. Modificada de: Mulry TJ, McIntyre LF. The Classification of Knee Meniscal Cartilage Tears. Operative Techniques in Sports Medicine. 2018;26(4):228-32.

\section{BIOMECÁ N I CA}

Sin duda, gran parte de lo que hoy conocemos acerca de los meniscos se lo debemos a los trabajos del Dr. Steven Arnoczky. ${ }^{18}$ Se sabe que soportan cargas, distribuyen las fuerzas, actúan como estabilizadores secundarios tanto AP como rotacional, aumentan la congruencia articular y están implicados en la propiocepción y la lubricación de la articulación. Es evidente el efecto condroprotector que ejercen, gracias al aro de tensión de sus fibras circunferenciales y a los puntos de anclaje en sus raíces. La ausencia o el deterioro de los mismos está asociado con daño condral irreversible y se ha demostrado que los pacientes con lesiones radiales mayores de $50 \%$ o con lesiones de la raíz posterior se comportan como pacientes meniscectomizados que van a progresar al deterioro del cartílago. 6,13,19,20

La literatura relaciona a las lesiones de la raíz posterior de los meniscos con el término "menisco incompetente", que es aquel estructuralmente sano, pero que pierde el aro de tensión al desprenderse de su raíz, y no es capaz de cumplir su función. Se ha demostrado que fijar las raíces devuelve al menisco su función y se recupera su efecto condroprotector.

\section{T R ATA M I EN TO}

Depende del estado del cartílago, los cambios degenerativos del menisco y la severidad y duración de los síntomas. Existen varias alternativas para enfocarlo: el tratamiento no quirúrgico está indicado cuando los síntomas son mínimos o la actividad demandante es escasa. Se recomienda a los pacientes cambiar de actividad, disminuir de peso, cambiar el calzado y en algunos casos ortesis con resultados variables. El tratamiento quirúrgico incluye meniscectomía parcial o total, reparo de la raíz o trasplante meniscal. 3,4,6,21,22

La meniscectomía parcial o total está indicada si la calidad del menisco no es lo suficientemente buena para soportar una sutura de tracción, cuando es imposible reducir el menisco al sitio original de la raíz o ante la presencia de cambios artrósicos. Otra indicación para no realizar un reparo de la raíz es que el paciente no quiera someterse al protocolo de rehabilitación. Habría que considerar como situación especial para reparar una raíz si el paciente es mal alineado con cartílago sano o con lesiones ligamentarias concomitantes, para lo cual se debe fijar la raíz con los procedimientos pertinentes según el caso. 
El escenario ideal para reparar una raíz es el paciente con lesión combinada del ligamento cruzado anterior o aquel con lesión aislada de la raíz de un menisco con cartílago sano. La edad no parece ser un factor determinante al momento de fijar una raíz meniscal, se deben tener en cuenta los demás parámetros como el estado del cartílago y del tejido a reparar.

\section{TÉCNICA QUIRÚR G ICA}

Recomendamos la técnica descrita por Harner y col. y destacamos la siguiente secuencia a seguir ${ }^{4}$ : a) identifique la lesión; b) escotaduroplastia reversa (menisco medial); c) prepare el área de inserción (cree área cruenta); d) paso de suturas de tracción a través del menisco (idealmente dos); e) túnel cruzando línea media y brocado; f) paso de suturas a través del túnel y g) fijación a 45 grados de flexión (figuras 8 a 10).

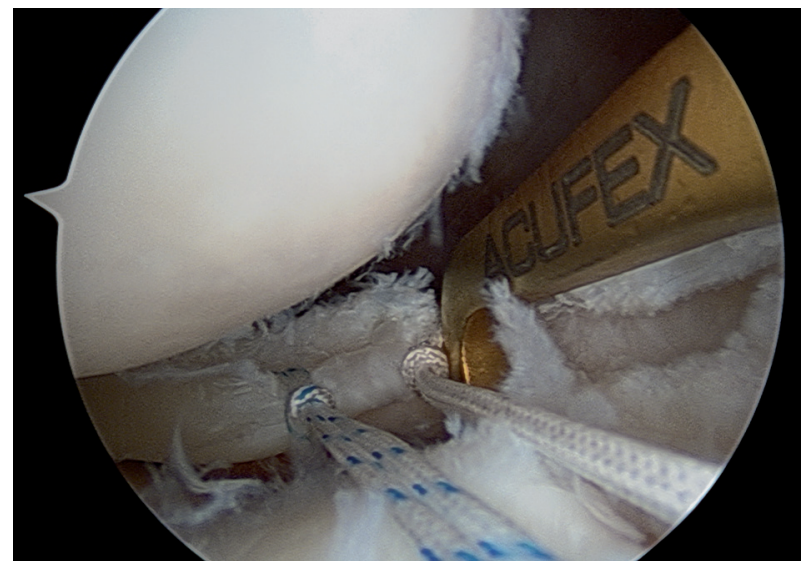

Figura 8. Suturas y guía para el túnel (menisco lateral, rodilla derecha).

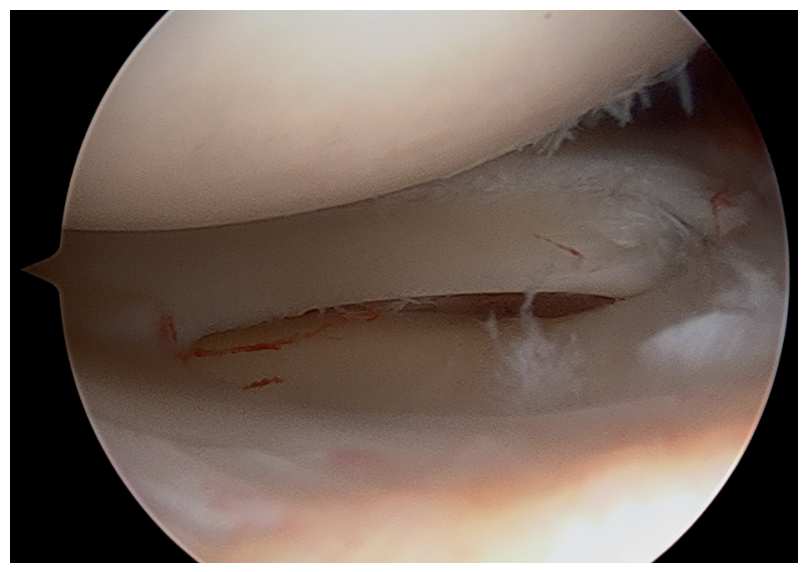

Figura 9. Raíz posterior del menisco lateral de la rodilla derecha reparada (nótese como se recupera el aro de tensión).

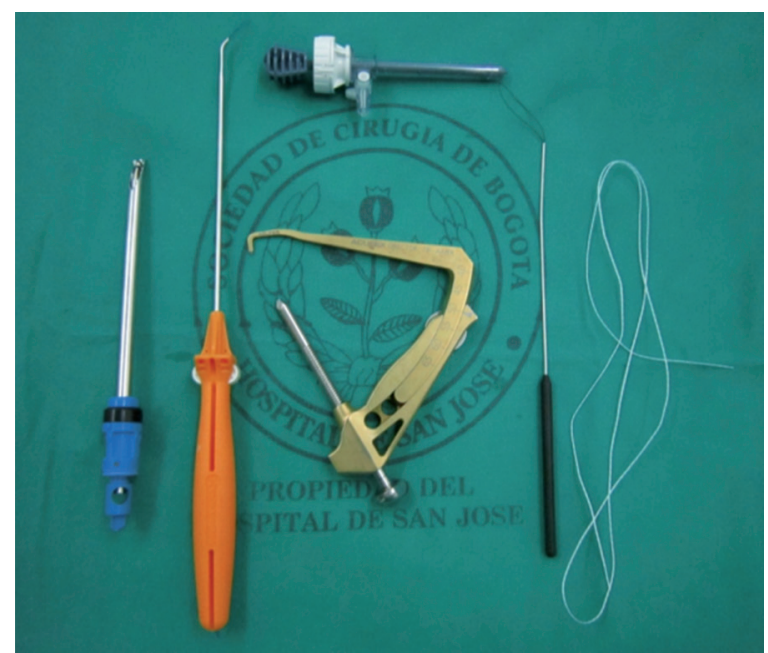

Figura 10. Instrumental requerido

Existen algunos aspectos desconocidos sobre los resultados biomecánicos luego de la artroscopia y el uso de la técnica de pull-out. En un modelo animal se encontró que si bien la técnica reduce la presión de contacto sobre el menisco, esta no llega a ser normal. ${ }^{23}$ Un estudio realizado en el laboratorio mostró que la aplicación de la técnica pull-out conduce a la reducción de la superficie de contacto óseo y mejora el perfil de transmisión de la carga al cartílago. ${ }^{24}$ Así mismo, la comparación entre variaciones de la técnica muestra un perfil biomecánico similar. ${ }^{25,26}$ La reparación mediante túneles transtibiales permite la estabilización de la presión de contacto y normalizar la función del menisco. ${ }^{27}$ Varios estudios han explorado la utilidad de la técnica de pull-out en la reparación de las lesiones de la raíz meniscal ${ }^{28-36}$ (tabla 1).

La escala Lysholm es utilizada a menudo en la evaluación de los efectos del tratamiento en los estudios que evalúan la eficacia de intervenciones para las lesiones de los meniscos; el instrumento ha sido validado en diferentes países. ${ }^{37} \mathrm{Su}$ fácil aplicación, así como su relación con aspectos de la calidad de vida, hacen que sea un instrumento utilizado en distintos centros del mundo. En esta serie de casos se han reportado valores de esta escala calificados como positivos o buenos para el paciente y similares a los reportes previos (tabla 1). Se ha encontrado una mejora significativa en la puntuación del dolor. La realización de estudios prospectivos y ensayos clínicos permitirá establecer con exactitud la eficacia clínica de la intervención. Sin embargo, estos resultados si bien no aportan el más alto nivel de evidencia científica posible, permiten crear el marco conceptual para desarrollar estudios futuros $\mathrm{y}$ entender el efecto terapéutico de la técnica quirúrgica. 
Tabla 1. Reportes de reparaciones de lesiones de raíz meniscal mediante la técnica de pull-out

\begin{tabular}{|c|c|c|c|c|}
\hline Autor & Autor & Tipo de estudio & Tipo de lesiones & Tipo de lesiones \\
\hline Eun y col. & 6 & Serie de casos & Lesión raíz posterior menisco medial & Lysholm previo 48,6 vs. 87,6 . \\
\hline LaPrade y col. & 49 & Estudio de cohorte retrospectiva & Lesión raíz posterior menisco medial o lateral & $\begin{array}{l}\text { Lysholm previo } 43 \text { vs. } 82 \\
\text { (menores de } 50 \text { años). } \\
\text { Lysholm previo } 63 \text { vs. } 79 \\
\text { (mayores de } 50 \text { años). }\end{array}$ \\
\hline Chung y col. & 40 & Estudio de cohorte prospectiva & Lesión raíz posterior menisco medial & Lysholm previo 52,1 vs. 83,8 . \\
\hline Cho y col. & 20 & Serie de casos & Lesión raíz posterior menisco medial & Lysholm previo 33,5 vs. 82,2 . \\
\hline Kim y col. & 30 & Estudio de cohorte retrospectiva & Lesión raíz posterior menisco medial & Lysholm previo 48,5 vs. 85,1 . \\
\hline Kim y col. & 23 & Estudio de tipo antes y después & Lesión raíz posterior menisco medial & Lysholm previo 54,3 vs. 92,5 . \\
\hline Lee y col. & 25 & Estudio de cohorte retrospectiva & Lesión raíz posterior menisco medial & Lysholm previo 57,4 vs. 87,6 . \\
\hline Lee y col. & 26 & Serie de casos & Lesión raíz posterior menisco medial & Lysholm previo 57 vs. 93,8. \\
\hline Moon y col. & 51 & Serie de casos & Lesión raíz posterior menisco medial & $\begin{array}{c}\text { Lysholm previo } 48,3 \text { vs. } 83,2 \text {. } \\
\text { Dolor pre } 7,4 \text { vs } 2,2\end{array}$ \\
\hline Seo y col. & 21 & Serie de casos & Lesión raíz posterior menisco medial & Lysholm previo 56,1 vs. 83. \\
\hline
\end{tabular}

\section{REH A B I L I TAC I Ó N}

Desde el posoperatorio inmediato se inicia la rehabilitación de este tipo de fijaciones. Se indican isométricos los cuádriceps y movilidad permanente del tobillo para estimular el retorno venoso, activando la musculatura de la pantorrilla. Los estudios realizados han demostrado que dos puntos de tracción son mejor que uno y permiten una recuperación acelerada de la movilidad de la rodilla. Se indican un par de muletas al menos durante 4 semanas para

\section{REFERENCIAS}

1. Pagnani MJ, Cooper DE, Warren RF. Extrusión of the medial meniscus. Arthroscopy. 1991;7(3):297-300.

2. Marzo JM, Kumar BA. Primary Repair of Medial Meniscal Avulsions. Am J Sports Med. 2007;35(8):1380-1383. doi:10.1177/0363546506297907.

3. Kenny C. Arthroscopic Repair of Avulsion of the Posterior Root and Body of the Lateral Meniscus: A Twenty-Year Follow-up. J Bone Jt Surgery-American Vol. 2009;91(12):2932-2936. doi:10.2106/ JBJS.H.01795.

4. Harner CD, Mauro CS, Lesniak BP, Romanowski JR. Biomechanical Consequences of a Tear of the Posterior Root of the Medial Meniscus. J Bone Jt Surgery-American Vol. 2009;91:257-270. doi:10.2106/JBJS.I.00500.

5. Marzo JM. Medial meniscus posterior horn avulsion. J Am Acad Orthop Surg. 2009;17(5):276-283.

6. Kim SB, Ha JK, Lee SW, et al. Medial Meniscus Root Tear Refixation: Comparison of Clinical, Radiologic, and Arthroscopic Findings With Medial Meniscectomy. Arthrosc J Arthrosc Relat Surg. 2011;27(3):346-354. doi:10.1016/j. arthro.2010.08.005. restringir el apoyo y a partir de la cuarta semana se van retirando hasta permitir el apoyo completo. Se autorizan ejercicios de bajo impacto alrededor del tercer mes y el retorno deportivo se da alrededor del sexto mes.

\section{CONFLICTO DE INTERESES}

El autor principal declara conflicto de intereses por ser consultor de Smith\&Nephew.

7. Hwang B-Y, Kim S-J, Lee S-W, et al. Risk Factors for Medial Meniscus Posterior Root Tear. Am J Sports Med. 2012;40(7):16061610. doi:10.1177/0363546512447792.

8. Matheny LM, Ockuly AC, Steadman JR, LaPrade RF. Posterior meniscus root tears: associated pathologies to assist as diagnostic tools. Knee Surgery, Sport Traumatol Arthrosc. 2015;23(10):31273131. doi:10.1007/s00167-014-3073-7.

9. Feucht MJ, Bigdon S, Bode G, et al. Associated tears of the lateral meniscus in anterior cruciate ligament injuries: risk factors for different tear patterns. J Orthop Surg Res. 2015;10(1):34. doi:10.1186/s13018-015-0184-x.

10. Lee SY, Jee W-H, Kim J-M. Radial Tear of the Medial Meniscal Root: Reliability and Accuracy of MRI for Diagnosis. Am J Roentgenol. 2008;191(1):81-85. doi:10.2214/AJR.07.2945.

11. Beasley, L, Robertson, D, Armfield D. Medial meniscus root tears: An unsolved problem-Demographic, radiographic, and arthroscopic findings. Pittsburgh Orthop J. 2005;16:155.

12. Berg EE. The meniscal ossicle: the consequence of a meniscal avulsion. Arthroscopy. 1991;7(2):241-243. http://www.ncbi.nlm. nih.gov/pubmed/2069638. 
13. Costa CR, Morrison WB, Carrino JA. Medial Meniscus Extrusion on Knee MRI: Is Extent Associated with Severity of Degeneration or Type of Tear? Am J Roentgenol. 2004;183(1):17-23. doi:10.2214/ ajr.183.1.1830017.

14. Brody JM, Lin HM, Hulstyn MJ, Tung GA. Lateral Meniscus Root Tear and Meniscus Extrusion with Anterior Cruciate Ligament Tear. Radiology. 2006;239(3):805-810. doi:10.1148/ radiol.2393050559.

15. Schnarkowski P, Tirman PF, Fuchigami KD, Crues J V, Butler MG, Genant HK. Meniscal ossicle: radiographic and MR imaging findings. Radiology. 1995;196(1):47-50. doi:10.1148/ radiology.196.1.7784586.

16. Jones A, Houang M, Low R, Wood D. Medial meniscus posterior root attachment injury and degeneration: MRI findings. Australas Radiol. 2006;50(4):306-313. doi:10.1111/j.14401673.2006.01586.x.

17. LaPrade CM, James EW, Cram TR, Feagin JA, Engebretsen L, LaPrade RF. Meniscal Root Tears. Am J Sports Med. 2015;43(2):363369. doi:10.1177/0363546514559684.

18. Choi C-J, Choi Y-J, Lee J-J, Choi C-H. Magnetic Resonance Imaging Evidence of Meniscal Extrusion in Medial Meniscus Posterior Root Tear. Arthrosc J Arthrosc Relat Surg. 2010;26(12):1602-1606. doi:10.1016/j.arthro.2010.05.004.

19. Berthiaume M-J. Meniscal tear and extrusion are strongly associated with progression of symptomatic knee osteoarthritis as assessed by quantitative magnetic resonance imaging. Ann Rheum Dis. 2005;64(4):556-563. doi:10.1136/ard.2004.023796.

20. Koenig JH, Ranawat AS, Umans HR, DiFelice GS. Meniscal Root Tears: Diagnosis and Treatment. Arthrosc J Arthrosc Relat Surg. 2009;25(9):1025-1032. doi:10.1016/j.arthro.2009.03.015.

21. Ahn JH, Wang JH, Yoo JC, Noh HK, Park JH. A pull out suture for transection of the posterior horn of the medial meniscus: using a posterior trans-septal portal. Knee Surgery, Sport Traumatol Arthrosc. 2007;15(12):1510-1513. doi:10.1007/s00167-007-0310-3.

22. Kim Y-M, Rhee K-J, Lee J-K, Hwang D-S, Yang J-Y, Kim S-J. Arthroscopic pullout repair of a complete radial tear of the tibial attachment site of the medial meniscus posterior horn. Arthroscopy. 2006;22(7):795.el-4. http://www.ncbi.nlm.nih.gov/ pubmed/16848058.

23. Seo J-H, Li G, Shetty GM, et al. Effect of Repair of Radial Tears at the Root of the Posterior Horn of the Medial Meniscus With the Pullout Suture Technique: A Biomechanical Study Using Porcine Knees. Arthrosc J Arthrosc Relat Surg. 2009;25(11):1281-1287. doi:10.1016/j.arthro.2009.05.014.

24. Padalecki JR, Jansson KS, Smith SD, et al. Biomechanical Consequences of a Complete Radial Tear Adjacent to the Medial Meniscus Posterior Root Attachment Site. Am J Sports Med. 2014;42(3):699-707. doi:10.1177/0363546513499314.

25. LaPrade CM, LaPrade MD, Turnbull TL, Wijdicks CA, LaPrade RF. Biomechanical Evaluation of the Transtibial Pull-Out Technique for Posterior Medial Meniscal Root Repairs Using 1 and 2
Transtibial Bone Tunnels. Am J Sports Med. 2015;43(4):899-904. doi:10.1177/0363546514563278.

26. Feucht MJ, Grande E, Brunhuber J, et al. Biomechanical Comparison Between Suture Anchor and Transtibial Pull-out Repair for Posterior Medial Meniscus Root Tears. Am J Sports Med. 2014;42(1):187-193. doi:10.1177/0363546513502946.

27. Chahla J, Moulton SG, LaPrade CM, Dean CS, LaPrade RF. Posterior Meniscal Root Repair: The Transtibial Double Tunnel Pullout Technique. Arthrosc Tech. 2016;5(2):e291-e296. doi:10.1016/j. eats.2016.01.006.

28. Eun SS, Lee SH, Sabal LA. Arthroscopic repair of the posterior root of the medial meniscus using knotless suture anchor: A technical note. Knee. 2016;23(4):740-743. doi:10.1016/j.knee.2016.03.001.

29. LaPrade RF, Matheny LM, Moulton SG, James EW, Dean CS. Posterior Meniscal Root Repairs: Outcomes of an Anatomic Transtibial Pull-Out Technique. Am J Sports Med. 2017;45(4):884891. doi:10.1177/0363546516673996.

30. Chung KS, Ha JK, Ra HJ, Kim JG. Prognostic Factors in the Midterm Results of Pullout Fixation for Posterior Root Tears of the Medial Meniscus. Arthrosc J Arthrosc Relat Surg. 2016;32(7):1319-1327. doi:10.1016/j.arthro.2015.12.046.

31. Cho J-H, Song J-G. Second-Look Arthroscopic Assessment and Clinical Results of Modified Pull-Out Suture for Posterior Root Tear of the Medial Meniscus. Knee Surg Relat Res. 2014;26(2):106113. doi:10.5792/ksrr.2014.26.2.106.

32. Kim J-H, Chung J-H, Lee D-H, Lee Y-S, Kim J-R, Ryu K-J. Arthroscopic Suture Anchor Repair Versus Pullout Suture Repair in Posterior Root Tear of the Medial Meniscus: A Prospective Comparison Study. Arthrosc J Arthrosc Relat Surg. 2011;27(12):1644-1653. doi:10.1016/j.arthro.2011.06.033.

33. Lee DW, Kim MK, Jang HS, Ha JK, Kim JG. Clinical and Radiologic Evaluation of Arthroscopic Medial Meniscus Root Tear Refixation: Comparison of the Modified Mason-Allen Stitch and Simple Stitches. Arthrosc J Arthrosc Relat Surg. 2014;30(11):1439-1446. doi:10.1016/j.arthro.2014.05.029.

34. Lee JH, Lim YJ, Kim KB, Kim KH, Song JH. Arthroscopic Pullout Suture Repair of Posterior Root Tear of the Medial Meniscus: Radiographic and Clinical Results With a 2-Year Follow-up. Arthrosc J Arthrosc Relat Surg. 2009;25(9):951-958. doi:10.1016/j. arthro.2009.03.018.

35. Moon H-K, Koh Y-G, Kim Y-C, Park Y-S, Jo S-B, Kwon S-K. Prognostic Factors of Arthroscopic Pull-out Repair for a Posterior Root Tear of the Medial Meniscus. Am J Sports Med. 2012;40(5):1138-1143. doi:10.1177/0363546511435622.

36. Seo H-S, Lee S-C, Jung K-A. Second-Look Arthroscopic Findings After Repairs of Posterior Root Tears of the Medial Meniscus. Am J Sports Med. 2011;39(1):99-107. doi:10.1177/0363546510382225.

37. Celik D, Coşkunsu D, Kılıçoğlu Ö. Translation and Cultural Adaptation of the Turkish Lysholm Knee Scale: Ease of Use, Validity, and Reliability. Clin Orthop Relat Res. 2013;471(8):26022610. doi:10.1007/s11999-013-3046-z. 